\title{
Using a hybrid approach to optimize experimental network design for aquifer parameter identification
}

\author{
Liang-Cheng Chang • Hone-Jay Chu • \\ Yu-Pin Lin • Yu-Wen Chen
}

Received: 16 October 2008 / Accepted: 18 August 2009

(C) Springer Science + Business Media B.V. 2009

\begin{abstract}
This research develops an optimum design model of groundwater network using genetic algorithm (GA) and modified Newton approach, based on the experimental design conception. The goal of experiment design is to minimize parameter uncertainty, represented by the covariance matrix determinant of estimated parameters. The design problem is constrained by a specified cost and solved by GA and a parameter identification model. The latter estimates optimum parameter value and its associated sensitivity matrices. The general problem is simplified into two classes of network design problems: an observation network design problem and a pumping network design problem. Results explore the relationship
\end{abstract}

L.-C. Chang · Y.-W. Chen

Department of Civil Engineering,

National Chiao Tung University,

Hsinchu, Taiwan, Republic of China

L.-C. Chang

e-mail: lcchang31938@gmail.com

Y.-W. Chen

e-mail: bsjacky@gmail.com

H.-J. Chu ( $\varangle)$ · Y.-P. Lin

Department of Bioenvironmental Systems

Engineering, National Taiwan University,

Taipei, Taiwan, Republic of China

e-mail: honejaychu@gmail.com

Y.-P. Lin

e-mail: yplin@ntu.edu.tw between the experimental design and the physical processes. The proposed model provides an alternative to solve optimization problems for groundwater experimental design.

Keywords Groundwater • Experimental design • Genetic algorithm

\section{Introduction}

In environmental monitoring such as groundwater level and quality investigations, the collected data may cause significant uncertainty, including extremely complicated variations in the observed values of measurable characteristics in space and time. Development of efficient procedures for designing and adjusting information-effective monitoring networks is an essential task for more accurately understanding the aquifer property. Therefore, these networks can be used to detect the aquifer property or to characterize groundwater resources for the management.

Evaluation of aquifer parameters (i.e., transmissivity and storage) has been a continuing field of research. Aquifer parameters varying with space are not easily identified. Experimental design is defined as the process of ensuring that a model provides good representation of a real system and identifies the aquifer parameters (Yeh 1986; Heidari and Ranjithan 1998; Hsu 
and Yeh 1989; Nishikawa and Yeh 1989; Carrera et al. 2005). That is, experimental design refers to the process of gathering information from measurements of what is modeled. Experimental conditions for design include the number and locations of pumping and observation wells, and observation frequency and duration (Hsu and Yeh 1989; Nishikawa and Yeh 1989). Systematically optimizing the design involves maximizing parameter estimate reliability, while minimizing experimental design cost is an important consideration. Hsu and Yeh (1989) formulated an experimental design problem as a general mathematical programming problem and demonstrate using a numerical example. They developed a model minimizing experimental design cost whose data yields parameter estimates with required reliability and formulated the experimental design problem as a nonlinear, mixed-integer programming problem. Since the variables are discrete, the gradient-based optimization technique fails. They adopted a heuristic approach to solve a mixed-integer programming problem and a modified Gauss-Newton algorithm to evaluate the design parameters. Nishikawa and Yeh (1989) developed a heuristic algorithm to minimize pumping test cost whose data yields parameter estimates of required reliability. Starting with an initial transmissivity vector, the algorithm sequentially identifies optimal designs, simulates the pumping test, estimates the unknown parameters, and repeats until transmissivity estimates converge. Those studies all formulated the problem as an experimental design problem but only proposed a heuristic method to solve the problem. Since the experimental problem is a nonlinear optimization problem and contains integer variables and a large number of control variables, developing an efficient and systematic algorithm to solve the problem remains a challenging task.

Over the last two decades, optimization techniques, including maximum likelihood method, genetic algorithm, tabu search, and simulated annealing, have been adopted to solve the ground water problem (Carrera and Neuman 1986; McKinney and Lin 1994; Zheng and Wang 1996; Wang and Zheng 1998; Tung and Chou 2004; Tsai et al. 2003; Tsai and Yeh 2004; Sidiropoulos and Tolikas 2004). Zheng and Wang (1996) applied two optimization approaches-tabu search and simulated annealing - to identify an optimal parameter structure in a one-dimensional aquifer. McKinney and Lin (1994) used genetic algorithm (GA) to solve groundwater management problems involving operation costs. Results revealed that GA can be used efficiently to solve complex groundwater management problems, as in well field development. Sidiropoulos and Tolikas (2004) used GA to minimize groundwater pumping cost, obtaining optimal well configuration and well discharge. Tsai et al. (2003) and Tsai and Yeh (2004) adopted the hybrid algorithm, which contains a GA, to solve the hierarchical optimization problem. First, GA searches for parameter structural patterns. Then, a quasi-Newton algorithm or Bayesian estimation procedure is used to iteratively improve parameter values. Accordingly, this study applies the GA and modified GaussNewton algorithm in the experimental design to solve a discrete, nonlinear, and optimal combinational problem. The GA easily determines the dimensions of a well network and the modified Gauss-Newton method estimates parameter values in an experimental design.

This study designs a regional pumping test network that minimizes estimated parameter uncertainty under a given budget. Results demonstrate that GA optimizes well network locations used in automated parameter estimation procedures. Additionally, this work explores the relationship between network location and physical processes.

\section{Mathematical formulation}

\section{Experimental network design}

This study designs a well network that minimizes estimated parameter uncertainty. Estimated parameter uncertainty is typically related to the covariance matrix:

$\operatorname{cov}(\hat{T}) \equiv E\left\{(T-\hat{T})^{\prime}(T-\hat{T})\right\}$

where $\hat{T}$ are the estimated parameters (transmissivity), $T$ are the true parameters, and $E$ represents the mathematical expectation. The 
covariance matrix of estimated parameters is approximately (Yeh and Yoon 1981)

$\operatorname{cov}(\hat{T})=\frac{F(\hat{T})}{M-L}\left[J_{D}^{\prime} J_{D}\right]^{-1}$

where

$F(\hat{T})$ the least square error in $\hat{T}$. A classical criterion for parameter estimation is the least square error. The objective function is defined as $F(\hat{T})=$ $\underset{T}{\operatorname{Min}} F(T)=\left[\left(h(\hat{T})-h^{0}\right)^{\prime}\left(h(\hat{T})-h^{0}\right)\right]$, where $h(\hat{T})$ is the vector of calculated hydraulic head based upon an estimated value of $\hat{T}$ and $h^{0}$ is the vector of observed hydraulic head.

$M \quad$ total number of observations

$L \quad$ total number of parameters

$J_{D} \quad$ Jacobian matrix of $h$ with respect to $T$, $\left.\left[\frac{\partial h_{i}}{\partial T_{j}}\right]\right|_{i=1, \ldots, M ; j=1, \ldots, L}$

The common criteria with least uncertainty for optimal design in groundwater design problems are A-optimality, D-optimality, and E-optimality. A-optimality refers to minimized covariance matrix trace. D-optimality is minimized covariance matrix determinant. E-optimality refers to minimized spectral radius. D-optimality is the most studied of all design criteria (Silvey 1980). The statistical D-optimality criterion for experimental design is herein adopted. The aim of the experimental design problem studied herein is to determine the number and location of observation and pumping wells, so that the design yields the least uncertain parameter estimates using D-optimality. The experimental design problem is formulated as:

$\underset{l o, l p, Q(l p)}{\operatorname{Min}}|\operatorname{cov}(\hat{T})|$

Subject to

$$
\begin{aligned}
& l o \subset \Omega_{o} \\
& l p \subset \Omega_{p} \\
& \text { num.(lo } \leq n o^{\max .} \\
& \text { num.(lp) } \leq n p^{\max }
\end{aligned}
$$

$Q(l p) \leq Q_{\max }$

$$
\begin{gathered}
F(\hat{T}(l o))=\operatorname{Min}_{T} \quad F(T \mid l o) \\
=\operatorname{Min}_{T}\left[\left(h-h^{0}\right)^{T}\left(h-h^{0}\right) \mid l o\right] \\
\frac{\partial}{\partial x}\left(T \frac{\partial h}{\partial x}\right)+\frac{\partial}{\partial y}\left(T \frac{\partial h}{\partial y}\right)+\frac{\partial}{\partial z}\left(T \frac{\partial h}{\partial z}\right) \\
-Q=S \frac{\partial h}{\partial t} \\
d(i, j) \geq \text { dis.(lo }), i \in l o, j \in l o, i \neq j \\
d(i, j) \geq \text { dis.(lp), } i \in l p, j \in l p, i \neq j
\end{gathered}
$$

where

num(lp) number of wells in pumping network num(lo) number of wells in observation network

lo set of observation network location

lp set of pumping network location

$\Omega_{o} \quad$ set of all candidate observation well locations

$\Omega_{p} \quad$ set of all candidate pumping well locations

$Q(l p) \quad$ pumping rate at pumping network

$d(i, j) \quad$ the distance between well $i$ and well $j$

$n o^{\text {max }}$ maximum number of observation wells

$n p^{\max } \quad$ maximum number of pumping wells

$Q_{\max } \quad$ maximum pumping rate

dis(lo) minimum distance between observation wells

dis $(l p) \quad$ minimum distance between pumping wells

Model constraints are given by Eqs. $4 a-4 i$, and with the following meanings: Eqs. $4 \mathrm{a}$ and $4 \mathrm{~b}$ define possible locations for observation and pumping wells in the candidate observation well locations $\left(\Omega_{o}\right)$ and pumping well locations $\left(\Omega_{p}\right)$. Equations $4 \mathrm{c}$ and $4 \mathrm{~d}$ give the maximum number of observation and pumping wells. Equation $4 \mathrm{e}$ limits the pumping rate. Equation $4 \mathrm{f}$ estimates parameters by minimizing least square errors. Equation $4 \mathrm{~g}$ is the groundwater flow equation for the hydraulic head response in an isotropic and confined aquifer using MODFLOW, where $h$ is the hydraulic head; $Q$ is the source/sink term; $S$ is the aquifer storage. The MODFLOW model was initially 
documented by McDonald and Harbaugh (1984). The MODFLOW is a physical finite-difference numerical flow model and a computer program developed by the US Geological Survey that numerically solves three-dimensional partial-differential equations for groundwater flow through a porous medium using a finitedifference method (McDonald and Harbaugh 1984; Harbaugh et al. 2000). In addition, Eqs. $4 \mathrm{~h}$ and $4 \mathrm{i}$ are the well distance constraints for considering the well density.

Observation and pumping network design

The general experimental design problem, given by Eqs. 3 and 4a, is split into two sub-problems for simplicity: an observation network design problem and a pumping network design problem.

1. Observation network design

The objective function is defined as

$$
\underset{\text { Mo }}{\operatorname{Min}}|\operatorname{cov}(\hat{T})|
$$

Subject to

lo $\subset \Omega_{o}$

num. $(l o) \leq n o^{\max }$

$$
\begin{aligned}
F(\hat{T}(l o)) & =\operatorname{Min}_{T} \quad F(T \mid l o) \\
& =\operatorname{Min}_{T} \quad\left[\left(h-h^{0}\right)^{T}\left(h-h^{0}\right) \mid l o\right]
\end{aligned}
$$

$$
\frac{\partial}{\partial x}\left(T \frac{\partial h}{\partial x}\right)+\frac{\partial}{\partial y}\left(T \frac{\partial h}{\partial y}\right)+\frac{\partial}{\partial z}\left(T \frac{\partial h}{\partial z}\right)-Q
$$$$
=S \frac{\partial h}{\partial t}
$$

$d(i, j) \geq \operatorname{dis.(lo),~} i \in l o, j \in l o, i \neq j$

2. Pumping network design

The objective function is defined as

$$
\underset{l p, Q(l p)}{\operatorname{Min}}|\operatorname{cov}(\hat{T})|
$$

\section{Subject to}

lp $\subset \Omega_{p}$

$$
Q(l p) \leq Q_{\max }
$$

$$
\text { num. }(l p) \leq n p^{\max }
$$

$$
\begin{aligned}
F(\hat{T}(l o)) & =\operatorname{Min}_{T} \quad F(T \mid l o) \\
& =\operatorname{Min}_{T} \quad\left[\left(h-h^{0}\right)^{T}\left(h-h^{0}\right) \mid l o\right]
\end{aligned}
$$

$$
\begin{aligned}
& \frac{\partial}{\partial x}\left(T \frac{\partial h}{\partial x}\right)+\frac{\partial}{\partial y}\left(T \frac{\partial h}{\partial y}\right)+\frac{\partial}{\partial z}\left(T \frac{\partial h}{\partial z}\right)-Q \\
& \quad=S \frac{\partial h}{\partial t}
\end{aligned}
$$

$d(i, j) \geq$ dis. (lp), $i \in l p, j \in l p, i \neq j$

\section{GA-based procedure}

Problems in the study are mixed-integer nonlinear time-varying problems with discontinuous variables (pumping/observation well locations) and continuous variables (estimated transmissivity), and cannot be solved by a single conventional optimization scheme. Therefore, this study further explores the problem structure and uses the hybrid algorithm to search for (1) where to construct an observation network or a pumping network and (2) estimated parameter values. The GAbased procedure determines the number and locations of observation and pumping wells in GA; then, the parameters are estimated for a given network design using the modified Gauss-Newton method with the least uncertainty. Figure 1 shows the conceptual design algorithm with the experiment design and parameter identification.

\section{Stage A: Encoding}

Analogous with biological systems, each chromosome bit is regarded as a gene and the string defines the genetic characteristics of an individual population. Decision variables encode to the binary string. The decision variables of these two problems differ. In problem 1, each chromosome bit represents candidate site location for an observation well. In problem 2 , the chromosome bit represents candidate site location for a pumping well. If the value of each chromosome bit equals one, then a well is located at the associated 


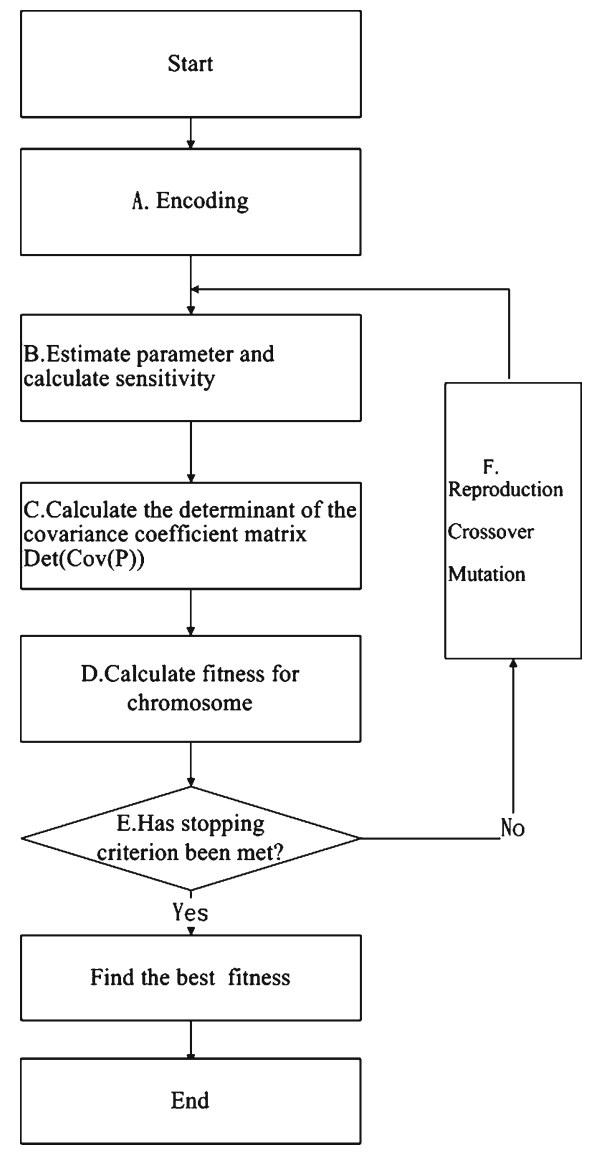

Fig. 1 Flowchart of design algorithm

candidate site. Otherwise, if the value of a bit is zero, then a well will not be installed.

Stage B: Estimate parameters and calculate sensitivity

After encoding the network design, the parameter (transmissivity) is estimated using UCODE (Poeter and Hill 1998). UCODE is a computer code for the universal inverse model. UCODE minimizes a weighted least-squares objective function with respect to parameter values using the modified Gauss-Newton method. This process is called parameter identification and is specified by Eqs. $4 \mathrm{f}$ and $4 \mathrm{~g}$.

This step determines sensitivity coefficients, which are elements of the Jacobian matrix. Sensitivity is the ratio of head difference to parameter perturbation. Parameter value differs slightly as it is perturbed from its unperturbed value.
Stage C: Calculate the covariance matrix determinant

This step calculates the covariance matrix determinant in Eq. 2.

Stage D: Calculate fitness for each chromosome

This step calculates the fitness of each chromosome. Fitness consists of the objective function and the penalty term if the decision variable is violated by the constraints (Hilton and Culver 2000).

Stage E: Has the stopping criteria been met?

This step checks the stopping criterion, based on the change in objective function and maximum generation. If the best design does not improve over 20 generations or a number of generations correspond to 100 generations, then the algorithm terminates.

Stage F: Reproduction, crossover, and mutation

The GA nucleus involves three operators-the reproduction operator, the crossover operator, and the mutation operator. If the model has not met the stopping criteria, then the parent generation undergoes reproduction, crossover, and mutation, generating the next generation of offspring (Goldberg 1989). The simple approach applying the solution operator, adopted in this

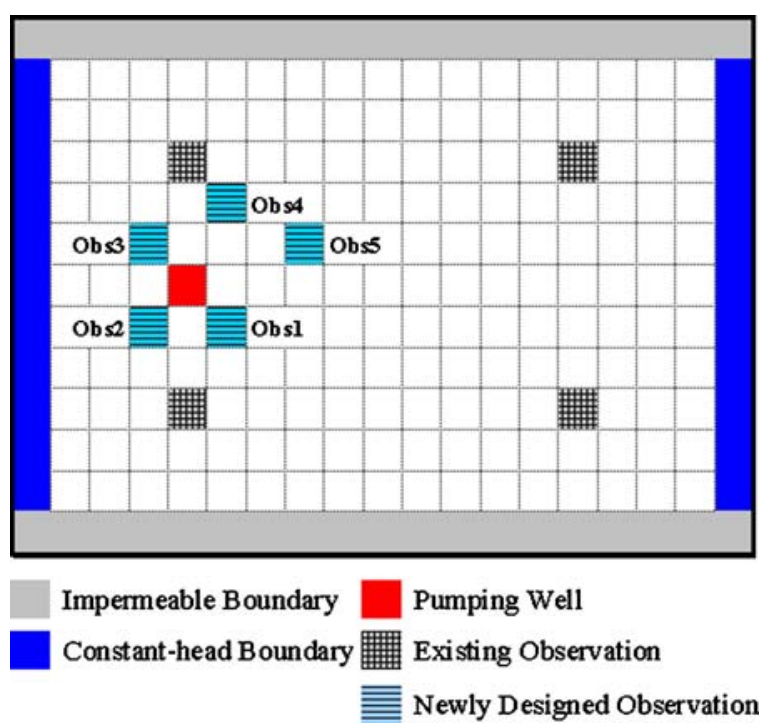

Fig. 2 Results in case 1 
Table 1 Location and sensitivity of the additional observation

\begin{tabular}{lllllll}
\hline & & Obs1 & Obs2 & Obs3 & Obs4 & Obs5 \\
\hline Case 1 & Location & $(6,8)$ & $(4,8)$ & $(4,6)$ & $(6,5)$ & $(8,7)$ \\
& Sensitivity $(\mathrm{s} / \mathrm{m})$ & 55.67 & 47.00 & 47.00 & 45.49 & 42.38 \\
Case 2 & Location & $(11,6)$ & $(11,8)$ & $(9,6)$ & $(8,8)$ & $(13,7)$ \\
& Sensitivity $(\mathrm{s} / \mathrm{m})$ & 72.57 & 72.57 & 72.53 & 60.15 & 50.80 \\
Case 3 & Location & $(14,8)$ & $(14,6)$ & $(16,8)$ & $(16,6)$ & $(12,7)$ \\
& Sensitivity $(\mathrm{s} / \mathrm{m})$ & 55.67 & 55.67 & 47.00 & 46.96 & 42.40 \\
\hline
\end{tabular}

study, involves tournament selection and uniform crossover.

This study selects a set of GA parameters based on previous studies (McKinney and Lin 1994; Wang and Zheng 1998; Chang et al. 2007) for performance analysis of the algorithm with various parameters and attempts finding suitable parameters. In the study, the GA parameters are as follows. Maximum number of generations in the program is 100 . Population size is 500 chromosomes. Rate of crossover is 0.8. Mutation probability is 0.01 .

\section{Numerical results and discussion}

Observation network design problem

Numerical analyses on a hypothetical case were performed to verify the effectiveness of the proposed methodology. A hypothetical, homogeneous, isotropic confined aquifer serves as an

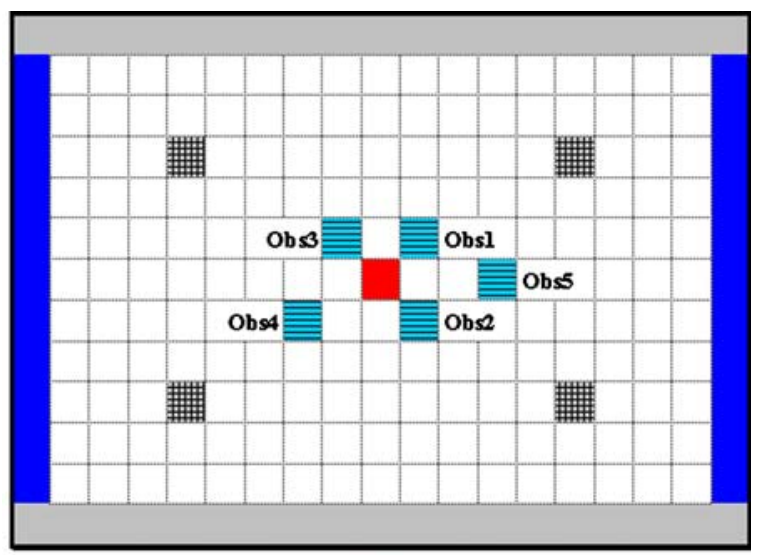

\section{Impermeable Boundary $\square$ Pumping Well \\ Constant-head Boundary 朖 Existing Observation}

Newly Designed Observation

Fig. 3 Results in case 2 example. Figure 2 presents the finite difference meshes with dimensions of $13 \times 19$. The mesh interval is equal to $500 \mathrm{~m}$. The example associates with no-flow boundary conditions to the north and south of the aquifer, and constant-head boundaries to the west and east with hydraulic heads of 50 and $20 \mathrm{~m}$. True value of transmissivity (T1) is assumed to be $3.0 \times 10^{-2} \mathrm{~m}^{2} / \mathrm{s}$. In problem 1 , the decision variable is the new observation location. The location of existing observation wells are $(5$, $4),(5,10),(15,4)$, and $(15,10)$. The origin $(0,0)$ is at the above left in the domain. The number of candidate observation wells is 183 . The model minimizes the estimated covariance while limiting fixed cost, with the number of newly designed observations limited to five. In addition, the minimum distance between two newly designed observations is $1,000 \mathrm{~m}$. Various pumping well locations are selected in the aquifer. The location of the existing pumping well is at $(5,7)$ in case $1,(10,7)$ in case 2 , and at $(15,7)$ in case 3 .
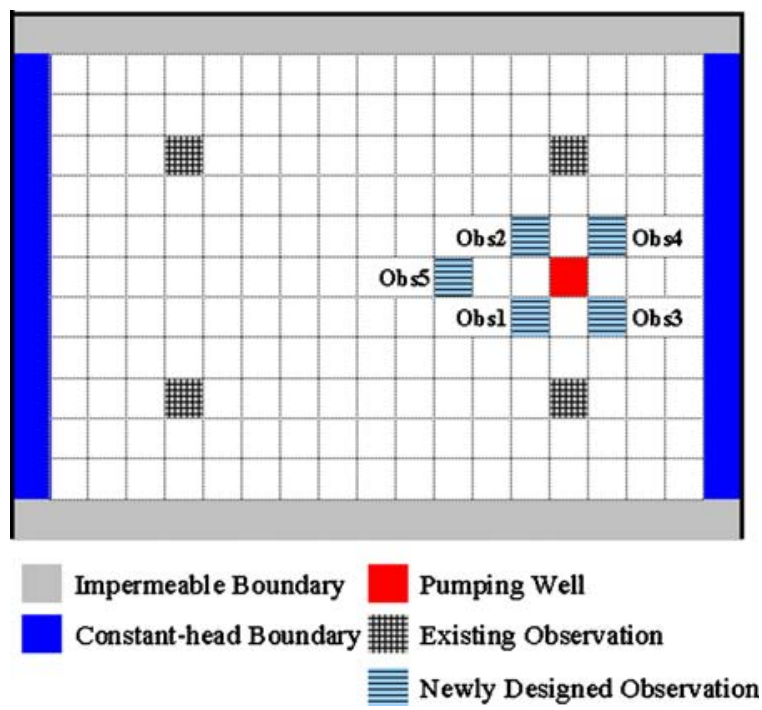

Fig. 4 Results in case 3 
Table 2 Optimal location of pumping well and estimated transmissivity

\begin{tabular}{lllllll}
\hline & $\begin{array}{l}\text { Optimal pumping } \\
\text { well location }\end{array}$ & $\begin{array}{l}\text { Pumping well at } \\
\mathrm{T} 1 \text { or T2 zone }\end{array}$ & $\begin{array}{l}\text { Estimated } \\
\mathrm{T} 1\left(\mathrm{~m}^{2} / \mathrm{s}\right)\end{array}$ & Relative error & $\begin{array}{l}\text { Estimated } \\
\mathrm{T} 2\left(\mathrm{~m}^{2} / \mathrm{s}\right)\end{array}$ & $\begin{array}{l}\text { Relative error } \\
\text { Case } 4\end{array}$ \\
Case 5 & $(4,9)$ & $\mathrm{T} 1$ & $1.98 \times 10^{-2}$ & $1.0 \%$ & $3.82 \times 10^{-2}$ & $4.5 \%$ \\
\hline
\end{tabular}

Table 1 presents the newly designed observations. The estimated parameter in the model is equal to the transmissivity (T1). In case 1 , the locations of the newly designed observation wells are $(4,6),(4,8),(6,5),(6,8)$ and $(8,7)$; in case 2 , they are $(8,8),(9,6),(11,6),(11,8)$, and $(13,7)$; in case 3 , they are $(12,7),(14,6),(14,8),(16,6)$, and $(16,8)$. Figures 2,3 , and 4 present the networks of observation network designs. Clearly, the newly designed observation network depends on pumping network locations. Table 1 also shows sensitivity in the newly designed observation (Obs1-Obs5). Results demonstrate that optimal observation location is most sensitive in all candidates. This finding reveals that the sensitivity decreases proportionally to the distance to the pumping well. Therefore, sensitivity increases with closer observation to the pumping well, reducing parameter evaluation uncertainty. The observation wells are firstly designed in the sensitive flow field to minimize uncertainty of estimated parameter values. The results confirm the previous study that the observation network for the transmissivity field is guided on the basis of the ar-

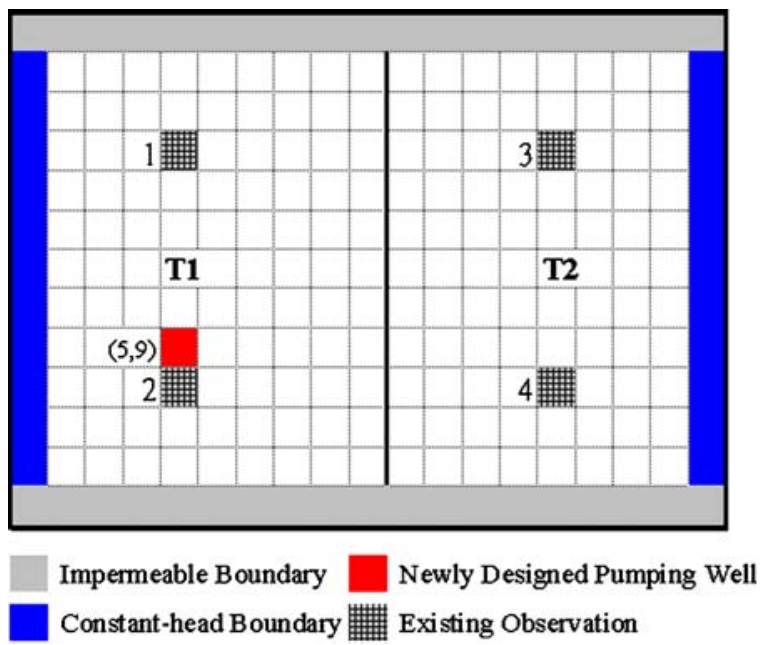

Fig. 5 Results in case 4 eas of the high hydraulic head gradient, since the information from the areas will lead to the most reliable management strategy (Andricevic 1990, 1993). The GA finds the optimal observation well effectively with various pumping well locations and is flexible in manipulating the highly complex spatial distribution. Moreover, when optimal observation network is determined, the information provided by the design is sufficient. The information is sufficient for parameter estimation so that the reliable parameters are identified (Chang et al. 2005).

Pumping network design problem

Following the above example, the decision variable in this design is only the pumping well location with 183 candidate sites. Existing observation wells are located at $(5,4),(5,10),(15,4)$, and $(15,10)$. Various numbers of pumping well designs demonstrate the cases. In the cases, case 4 designs a single pumping well, while case 5 designs two pumping wells. The pumping rate for each well is assumed to be $0.5 \mathrm{~m}^{3} / \mathrm{s}$.

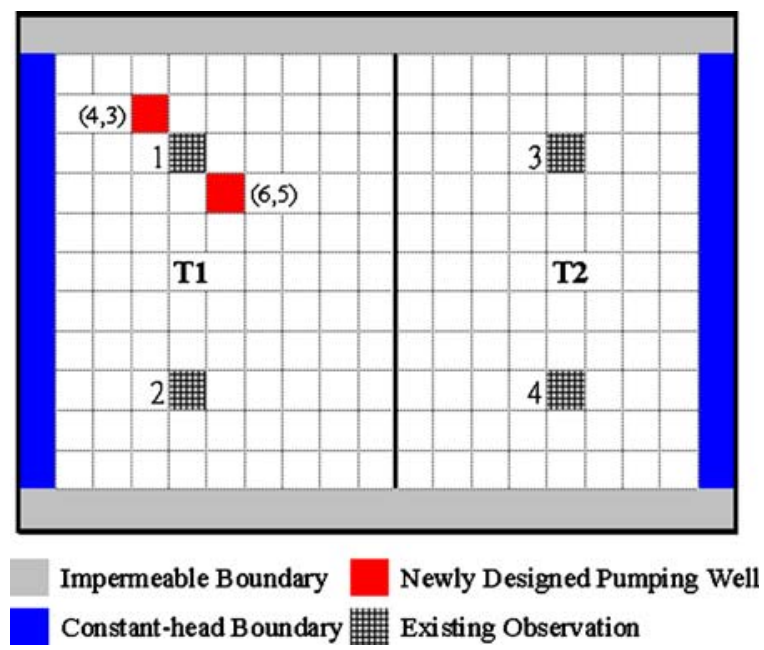

Fig. 6 Results in case 5 


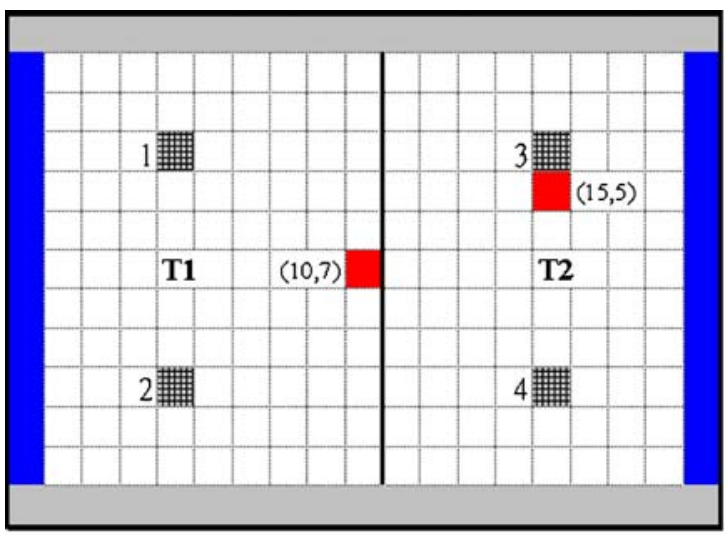

Impermeable Boundary

Constant-head Boundary 浀 Existing Observation

Fig. 7 Plan view in cases $6 b$ and $6 c$

Table 2 lists case conditions and optimization results. The optimal pumping location obtains parameter vales (T1, T2), as presented in Table 2 below. Transmissivity in case 4 is estimated as $\mathrm{T} 1=1.98 \times 10^{-2}$ and $\mathrm{T} 2=3.82 \times 10^{-2} \mathrm{~m}^{2} / \mathrm{s}$ by UCODE, with relative errors of $1 \%$ and $4.5 \%$. Estimated transmissivity in case 5 is $\mathrm{T} 1=$ $1.98 \times 10^{-2}$ and $\mathrm{T} 2=3.84 \times 10^{-2} \mathrm{~m}^{2} / \mathrm{s}$ and relative errors are $1 \%$ and $4 \%$. Results demonstrate that the optimal pumping well location in case 4 is $(5,9)$, as presented in Fig. 5. Optimal pumping well locations in case 5 are at $(4,3)$ and $(6,5)$ in Fig. 6. Results also indicate that the optimal well locations in both cases are in the zone T1. Results reveal that the pumping network is sensitive to geological conditions. Pumping wells are designed in the sensitive geological zone to increase experimental design reliability. Thus, the network design provides the sufficient information for reliable estimation when the network is firstly located in the sensitive geological zone (Chang et al. 2005). Furthermore, the required average CPU time for above cases is $16,308 \mathrm{~s}(4.5 \mathrm{~h})$ on AMD CPU (K7-1 GHz).

This work compares parameters estimated by the optimal pumping network with those estimated by other pumping networks to verify that the optimal pumping network obtained in case 4 is an efficient network design for estimating practical-situation parameters. The pumping site of case 6 a locates at $(5,9)$ following case 4 (Fig. 5). Pumping wells in cases $6 \mathrm{~b}$ and $6 \mathrm{c}$ are randomly chosen at $(10,7)$ and $(15,5)$, as presented in Fig. 7. This work obtains the observed hydraulic head estimating the parameters in these cases by adding the simulated head to the head noise generated at the observation sites. The head noise is generated using standard normal distribution with zero mean and standard deviation of 0.1 . The observation data are then used to estimate parameters using UCODE.

Table 3 presents the estimated parameters for the validation. In case $6 \mathrm{a}$, the relative error when compared to true parameter values is $3.0 \%$ in optimal pumping network. In case $6 \mathrm{~b}$, the relative errors are $48.5 \%$ and $43.5 \%$. In case $6 c$, the relative errors are $13.5 \%$ and $12.5 \%$. A comparison of relative errors reveals that, among three cases, case 6 a has the lowest estimation error. This result reveals that the design using GA is substantially better and transmissivity is accurately estimated based on effective information by applying the GA. Furthermore, by the two-level formulation, the discrete nature of the original problem is considered in the problem and facilitates the application of other computational efficient algorithms to solve the parameter estimation problem (sub-problem) and thus reduce the computational burden. This study uses the GA to solve the main problem and the GA accomplishes the discrete nature of searching for optimal well network alternatives. Within the GA, each chromosome

Table 3 Estimated T1 and T2 under various pumping locations

\begin{tabular}{llllrr}
\hline Case & $\begin{array}{l}\text { Location of } \\
\text { pumping well }\end{array}$ & $\begin{array}{l}\text { Estimated } \\
\mathrm{T} 1\left(\mathrm{~m}^{2} / \mathrm{s}\right)\end{array}$ & Relative error & \multicolumn{2}{l}{$\begin{array}{l}\text { Estimated } \\
\mathrm{T} 2\left(\mathrm{~m}^{2} / \mathrm{s}\right)\end{array}$} \\
\hline Case 6a & $(5,9)^{*}$ & $2.06 \times 10^{-2}$ & $3.0 \%$ & $4.12 \times 10^{-2}$ & $3.0 \%$ \\
Case 6b & $(10,7)$ & $1.03 \times 10^{-2}$ & $48.5 \%$ & $2.26 \times 10^{-2}$ & $43.5 \%$ \\
Case 6c & $(15,5)$ & $1.73 \times 10^{-2}$ & $13.5 \%$ & $3.52 \times 10^{-2}$ & $12.0 \%$ \\
\hline
\end{tabular}

*represents the optimal design case 
(network design) associates a sub-problem which contains nonlinear characteristics. The GA is more effective in finding a nearly global optimal solution in the problem with integer variables when compared to conventional gradient-based optimization methods (Sciortino et al. 2002).

\section{Conclusions}

This study explores mathematical characteristics of the regional groundwater network design and reformulates it as a two-level optimization problem to facilitate the GA and modified Gauss-Newton algorithm application. Based on the formulation, discrete decision variables of the problem are solved in the main-problem using the GA, while continuous decision variables of the problem are computed in the parameter estimation problem using the modified GaussNewton algorithm. The methodology is developed to minimize uncertainty of the estimated aquifer parameters (transmissivity) by selecting optimal locations of the observation or pumping network. The GA determines the number and locations of observation and pumping wells; then, the parameters are estimated for a given network design using Gauss-Newton algorithm.

Results indicate that GA successfully solves the experimental design problem, confirmed in numerical experiments in which the proposed method is computationally efficient. This study adopts this fundamental approach to demonstrate that the design yields a reliable transmissivity value. This approach further investigates two points. First, observation wells are designed close to the sensitive flow field to minimize uncertainty of estimated parameter values. Second, locating pumping wells in the least transmissivity zone ensures much sensitivity, and effectively designs pumping wells in the least transmissivity zone. Other suggestions follow.

The traditional experimental design is a sequential design. The optimal design is found only for each stage, not for all stages. The multi-stage design is favorable for long-term groundwater planning. The design policies in total stages should be regarded as experimental design guidelines.
A complete regional field investigation of aquifer parameters should include two main stages, regional network planning and on-site pumping test design. The most rational approach integrates the sampling design procedure into an experimental design. Future studies could further study observation frequency and duration. This work recommends that future studies clarify the relationship between total cost and transmissivity uncertainty to determine a trade-off between the two.

Acknowledgements The authors would like to acknowledge Hsin-Hua Chen who have contributed to the programming and the implement. In addition, we would like to thank the anonymous reviewers, editors and helpers for their contributions to this study.

\section{References}

Andricevic, R. (1990). A real-time approach to management and monitoring of groundwater hydraulic. Water Resources Research, 26(11), 2747-2755.

Andricevic, R. (1993). Coupled withdrawal and sampling designs for groundwater supply models. Water Resources Research, 29(1), 5-16.

Carrera, J., \& Neuman, S. (1986). Estimation of aquifer parameters under transient and steady state conditions: Maximum likelihood method incorporating prior information. Water Resources Research, 22-2, 199-210.

Carrera, J., Alcolea, A., Medina, A., Hidalgo, J., \& Slooten, L. J. (2005). Inverse problem in hydrogeology. Hydrogeology Journal, 13, 206-222.

Chang, L. F., Sun, N. Z., \& Yeh, W. W.-G. (2005). Optimal observation network design for parameter structure identification in groundwater modeling. Water Resources Research, 41(3), W03002.

Chang, L. C., Chu, H. J., \& Hsiao, C. T. (2007). Optimal planning of a dynamic pump-treat-inject groundwater remediation system. Journal of Hydrology, 342(3-4), 295-304.

Goldberg, D. E. (1989). Genetic algorithms in search, optimization, and machine learning. Reading: AddisonWesley.

Harbaugh, A. W., Banta, E. B., Hill, M. C., \& McDonald, G. (2000). MODFLOW-2000, the U.S. Geological Survey Modular Ground-Water Model-user guide to modularization concepts and the ground-water flow process. US Geological Survey.

Heidari, M., \& Ranjithan, S. R. (1998). A hybrid optimization approach to the estimation of distributed parameters in two-dimensional confined aquifers. Journal of the American Water Resources Association, 34(4), 909920.

Hilton, A. B. C., \& Culver, T. B. (2000). Constraint handling for genetic algorithms in optimal remediation 
design. Journal of Water Resources Planning and Management, 126(3), 128-137.

Hsu, N. S., \& Yeh, W. W.-G. (1989). Optimum experimental design for parameter identification in groundwater hydrology. Water Resources Research, 25(5), 1025-1040.

McDonald, M. G., \& Harbaugh, A. W. (1984). A modular three-dimensional finite difference ground-water flow model. Reston: US Geological Survey.

McKinney, D. C., \& Lin, M. D. (1994). Genetic algorithm solution of groundwater management models. Water Resources Research, 30(6), 1897-1906.

Nishikawa, T., \& Yeh, W. W.-G. (1989). Optimal pumping test design for the parameter identification of groundwater system. Water Resources Research, 25(7), 17371747.

Poeter, E. P., \& Hill, M. C. (1998). Documentation of UCODE, a computer code for universal inverse modeling. U.S. Geological Survey Open-File Report 984080 .

Sciortino, A., Harmon, T. -C., \& Yeh, W. W.-G. (2002). Experimental design and model parameter estimation for location a dissolving dense nonaqueous phase liquid pool in groundwater. Water Resources Research, 38(5), 1057.

Sidiropoulos, E., \& Tolikas, P. (2004). Well location and constraint handling in groundwater pumping cost minimization via genetic algorithm. Water, Air, and Soil Pollution Focus, 4, 227-239.
Silvey, S. D. (1980). Optimal design - an introduction to the theory for parameter estimation. New York: Chapman and Hall.

Tsai, F. T. C., \& Yeh, W. W. G. (2004). Characterization and identification of aquifer heterogeneity with generalized parameterization. Water Resources Research, 40(10), W10102.

Tsai, F. T. C., Sun, N. Z., \& Yeh, W. W. G. (2003). A combinatorial optimization scheme for parameter structure identification in ground water modeling. Ground $\mathrm{Wa}$ ter, 41(2), 156-169.

Tung, C. P., \& Chou, C. A. (2004). Pattern classification using tabu search to identify the spatial distribution of groundwater pumping. Hydrogeology Journal, 12, 488-496.

Wang, M., \& Zheng, C. (1998). Groundwater management optimization using genetic algorithms and simulated annealing: Formulation and comparison. Journal of the American Water Resources Association, 34(3), 519530.

Yeh, W. W.-G. (1986). Review of parameter identification procedures in ground-water hydrology-the inverse problem. Water Resources Research, 22(2), 95-108.

Yeh, W. W.-G., \& Yoon, Y. S. (1981). Parameter identification with optimal dimension in parameterization. Water Resources Research, 17(3), 664-672.

Zheng, C., \& Wang, P. (1996). Parameter structure identification using tabu search and simulated annealing. Advances in Water Resources, 19(4), 215-224. 